\title{
Physiological Response of Escherichia coli W3110 and BL21 to the Aerobic Expression of Vitreoscilla Hemoglobin
}

\author{
Alvaro R. Lara*, Janet Galindo, Karim E. Jaén, Mariana Juárez, and Juan-Carlos Sigala \\ Departamento de Procesos y Tecnología, Universidad Autónoma Metropolitana-Cuajimalpa, Vasco de Quiroga \\ 4871, Santa Fe, CP 05348, Mexico City, Mexico

\begin{abstract}
The aerobic growth and metabolic performance of Escherichia coli strains BL21 and W3110 were studied when the Vitreoscilla hemoglobin ( $\mathrm{VHb}$ ) was constitutively expressed in the chromosome. When VHb was expressed, acetate production decreased in both strains and was nearly eliminated in BL21. Transcriptional levels of the glyoxylate shunt genes decreased in both strains when VHb was expressed. However, higher transcription of the $\alpha$-ketoglutarate dehydrogenase genes were observed for W3110, while for BL21 transcription levels decreased. VHb expression reduced the transcription of the cytochrome $\mathrm{bo}_{3}$ genes only in BL21. These results are useful for better selecting a production host.
\end{abstract}

Keywords: Vitreoscilla hemoglobin, aerobic cultures, E. coli W3110, BL21, transcriptional analysis

Received: April 16, 2020 Accepted: July 13, 2020

First published online: July 14,2020

*Corresponding author Phone: $+52-55-58146500$ Fax: +52-55-58146500 E-mail: alara@cua.uam.mx

Supplementary data for this paper are available on-line only at http://jmb.or.kr.

PISSN 1017-7825 elSSN 1738-8872

Copyright(C) 2020 by The Korean Society for Microbiology and Biotechnology
A well-established technology to cope with $\mathrm{O}_{2}$ limitation is the expression of Vitreoscilla hemoglobin (VHb). Possible actions of $\mathrm{VHb}$ are the binding to $\mathrm{O}_{2}$ and its delivery to the respiratory chain [1-3]. However, the effect of $\mathrm{VHb}$ under aerobic conditions has been only scarcely studied. The VHb-enhanced respiratory activity may increase the fluxes in the tricarboxylic acid cycle (TCA), lowering the aerobic acetate production [4]. As a result, aerobic $\mathrm{VHb}$ expression reduced acetate production in several Escherichia coli strains [4], lactate production by $\mathrm{CHO}$ cells [5], and the lipids production in Yarrowia lipolytica [6]. The impact of VHb expression in aerobic cultures of the strains E. coli W3110 and BL21, relevant for industrial and academic purposes [7, 8], was studied here. To avoid gene copy variations arising from plasmid-based expression, a single vgb gene copy (coding for $\mathrm{VHb}$ ) was inserted in the chromosome of BL21 and W3110 and constitutively expressed. The effect of VHb expression was studied in batch cultures (Fig. 1). W3110 produced acetate concomitantly with biomass formation and glucose consumption. At the point of glucose depletion, acetate concentration, oxygen transfer (OTR) and carbon dioxide evolution (CER) rates reached their maximum (Fig. 1C). OTR and CER rapidly decrease thereafter, indicating no $\mathrm{O}_{2}$ limitations. Then, both rates increased transiently, coincident with acetate reassimilation (Fig. 1A). When $\mathrm{VHb}$ was expressed in W3110, the profiles were similar (Figs. 1B and 1D), but around 30\% less acetate was accumulated, in comparison with the non-expressing strain. In the case of strain BL21, low amounts of acetate were accumulated (Fig. 1E). Accordingly, no second peaks of OTR and CER were detected after glucose depletion (Fig. 1G). Strain BL21 is well known for its low acetate accumulation and is amply used for high cell-density processes [10]. The low acetate production by BL21 has been attributed to a more active higher expression of glyoxylate shunt genes, compared with K12-derived strains [10, 11]. When VHb was expressed in BL21, acetate accumulation was nearly eliminated (Fig. 1F). Minor amounts of lactate and succinate were found in the samples.

Despite using the same expression system, the active $\mathrm{VHb}$ concentration was more than three-fold higher for BL21 than for W3110 (Fig. 2), which may be attributed to a lower protease activity in BL21, compared to W3110 [7]. The amounts of active $\mathrm{VHb}$ found are small compared to previous reports that range from $13-238 \mathrm{nmol} \mathrm{g}_{\mathrm{wcw}}{ }^{-1}$ [11] to 3.4-3.8 mmol g $\mathrm{DCW}^{-1}$ (ca. 850-950 $\mathrm{nmol} \mathrm{g}_{\mathrm{WCW}^{-1}}{ }^{-1}$ [12] using high-copy number plasmids. Nevertheless, the comparatively small amount of $\mathrm{VHb}$ caused relevant physiological changes, as detailed below. While $\mathrm{VHb}$ expression did not affect the specific growth rate $(\mu)$ in W3110, it was ca. $15 \%$ lower in BL21 $v g b^{+}$, compared to its parent strain (Table 1). The acetate yields decreased by 30 and $60 \%$ in W3110 and BL21, respectively, when VHb was expressed. The specific glucose uptake rate $\left(q_{s}\right)$ was $c a$. 12\% higher in BL21 than in W3110 wild-types (Table 1). Marisch et al., [7] demonstrated that strain BL21 displays a higher expression of genes involved in glucose transport than K-12 strains. Namely, genes coding for the galactiol PTS permease (gat $A B C$ ), mannose PTS permease (manXYZ), maltose ABC transporter (malKFGE), and LamB porin (lamB) were overexpressed in BL21, in comparison with a K12 derived strain [7]. This can contribute to the higher $q_{S}$ observed for BL21, compared to W3110. When $\mathrm{VHb}$ was expressed in $\mathrm{W} 3110, q_{S}$ increased by $19 \%$, compared to the wild-type (Table 1). This may point to an increased glucose oxidation capacity. Growth rate reduction, together with 


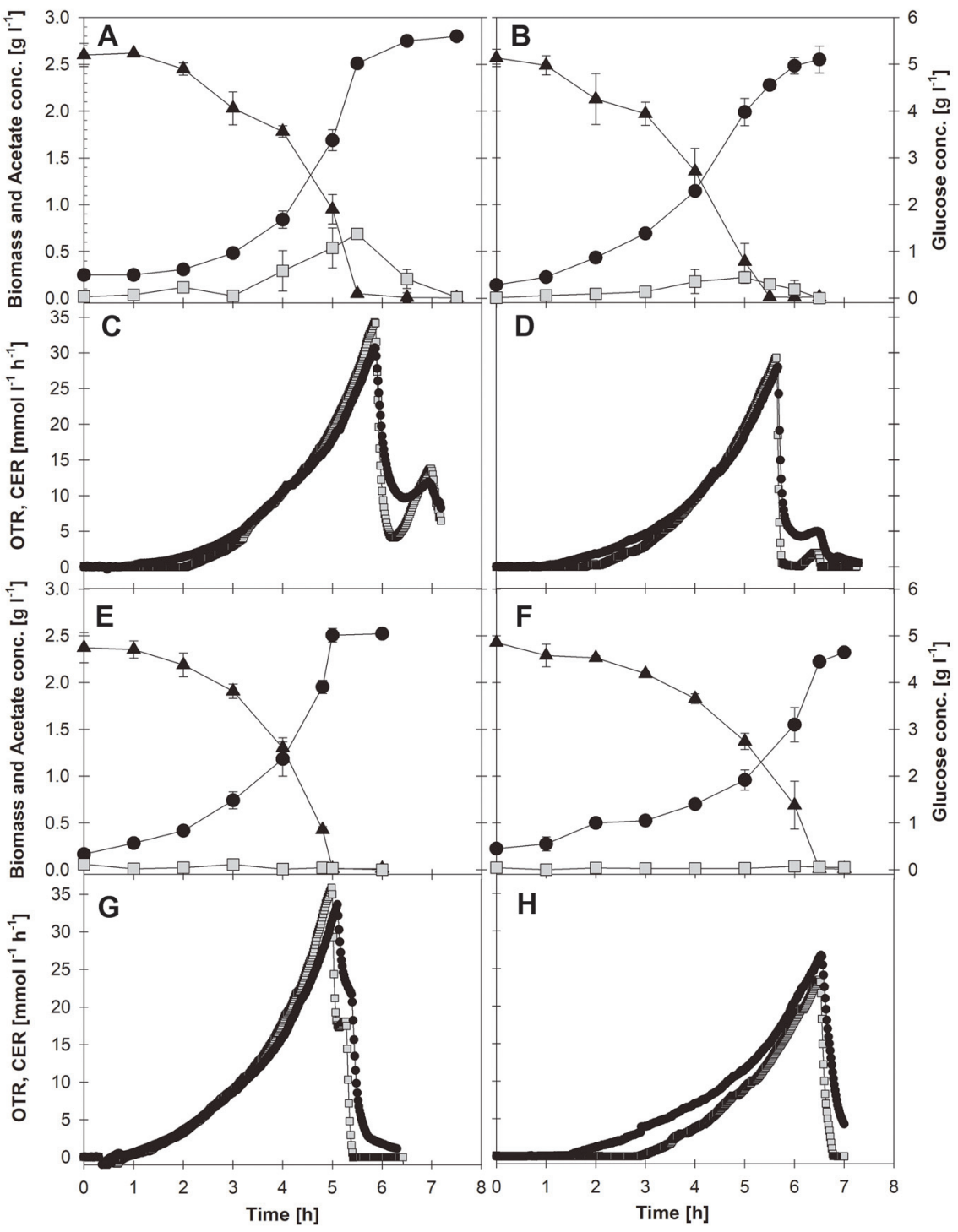

Fig. 1. Growth profiles of the engineered $E$. coli strains. A: Cell growth (circles), glucose (triangles) and acetate concentration (squares) in cultures of strain W3110 recA; B: Cell growth (circles), glucose (triangles) and acetate concentration (squares) in cultures of strain W3110 $\mathrm{recA}^{-} \mathrm{vgb}^{+}$; C: Oxygen transfer rate (OTR, squares) and carbon dioxide evolution rate (CER, circles) in cultures of strain W3110 recA; C: Oxygen transfer rate (OTR, squares) and carbon dioxide evolution rate (CER, circles) in cultures of strain W3110 recA $\mathrm{vgb}^{+}$; E: Cell growth (circles), glucose (triangles) and acetate concentration (squares) in cultures of strain BL21 recA; F: Cell growth (circles), glucose (triangles) and acetate concentration (squares) in cultures of strain BL21 recA $v g b^{+}$; G: Oxygen transfer rate (OTR, squares) and carbon dioxide evolution rate (CER, circles) in cultures of strain BL21 recA; H: Oxygen transfer rate (OTR, squares) and carbon dioxide evolution rate (CER, circles) in cultures of strain BL21 recA- $v g b^{+}$. Cultures were carried out in mineral medium supplemented with $5 \mathrm{~g} \mathrm{l}^{-1}$ of glucose under aerobic conditions (DOT $\geq 30 \%$ air sat.) and $\mathrm{pH} 7.0$ in 11 bioreactors. Vertical lines represent the experimental deviation between triplicate cultures.

increased $q_{S}$ and $q_{\mathrm{O} 2}$ have been also reported for evolved K-12 derivatives [14]. A slight $q_{s}$ decrease (ca. 8\%) was observed when $\mathrm{VHb}$ was expressed in strain BL21. Such a small decrease $q_{s}$ under aerobic conditions is in agreement with previous results expressing $\mathrm{VHb}$ from a high copy-number phagemid under aerobic conditions [4]. Other authors reported a $27 \%$ decrease of $q_{s}$ when $\mathrm{VHb}$ is expressed from a plasmid in strain MG1655 during microaerobic fed-batch cultures [15]. The $q_{s}$ decrease observed only in BL21 could be linked to the higher VHb expression, compared to $\mathrm{W} 3110$, and growth rate reduction. The specific rate of acetate production $\left(q_{\text {acet }}\right)$ decreased in the strains expressing VHb. Nonetheless, the specific rate of lactate production, which was unaffected by VHb in W3110, increased significantly when VHb was present in BL21. Lactate production under aerobic conditions has been reported for BL21 and attributed to overflow metabolism (excretion of partially oxidized carbon molecules under aerobic conditions) $[7,16]$. When aerobic acetate production was diminished 


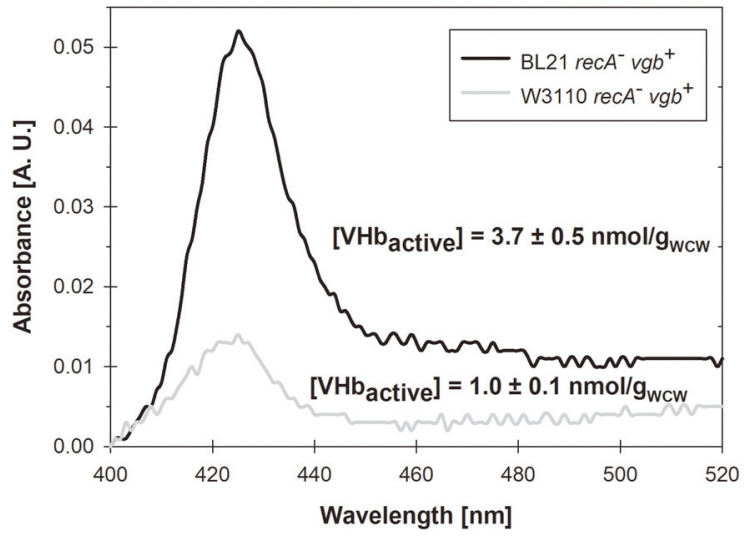

Fig. 2. Typical CO differential spectra of E. coli strains BL21 (upper line) and W3110 (lower line) constitutively expressing VHb. Samples were taken from aerobic cultures and spectral analyses were performed from the lysate of $0.5 \mathrm{~g}$ of wet cells. Average and standard deviation includes the results from triplicate cultures.

through inactivation of the involved pathways, lactate production increased [16], coincident with the results of Table 1. The effect of $\mathrm{VHb}$ on specific rate of succinate production was negligible for W3110 but in BL21 decreased by half. The specific oxygen uptake $\left(q_{\mathrm{O}_{2}}\right)$ and $\mathrm{CO}_{2}$ production $\left(q_{\mathrm{CO}_{2}}\right)$ rates in W3110 increased by $20 \%$ when $\mathrm{VHb}$ was expressed, in agreement with its hypothetic function. The estimated $q_{\text {ATP }}$, directly proportional to $q_{02}$, also indicates a higher energy generation rate in $\mathrm{W} 3110 \mathrm{vg} b^{+}$and BL21 $\mathrm{vg} b^{-}$, compared with W3110 $\mathrm{vg} b^{-}$and BL21 $v g b^{+}$, respectively.

The transcription level of $z w f$ gene, coding for the first enzyme of the pentose phosphate pathway (PPP) was nearly two-fold in W3110vgb $b^{+}$, but was slightly lower in BL21 $v g b^{+}$, compared with their parent strains (Fig. 3). This may indicate a change in the flux to the PPP, since the expression of $z w f$ is consistently linked to the flux in the corresponding pathway [16]. This is consistent with flux balance analysis performed by Tsai and coworkers [12], who estimated a higher flux through the PPP when VHb was expressed in strain W3110 under microaerobic conditions. However, a similar hypothesis about the flux through the PPP in BL21 cannot be formulated with the available information. Nevertheless, it is known that $z w f$ expression is growth-rate regulated [17], which is in agreement with the lower $\mu$ in BL21 $v g b^{+}$. The acetate forming pathways were slightly affected by the presence of $\mathrm{VHb}$ in W3110. While the poxB expression was unaffected in both strains, pta expression in BL21 was slightly repressed. This is in agreement with the observed reduction of acetate production. Genes from the glyoxylate shunt in BL21 were also slightly repressed when $\mathrm{VHb}$ was present. This may be linked to a lower re-assimilation of acetate during cell growth, consequence of a lower acetate production. The genes coding for enzymes of the oxidative decarboxylation of $\alpha$-ketoglutarate $(s u c A$ and $s u c B)$ were overexpressed in $\mathrm{W} 3110 \mathrm{vgb}^{+}$, relative to its wild-type. sucA transcription level is also consistently linked to the flux in the involved reaction [18]. The reaction involved produces $\mathrm{CO}_{2}$, which is in agreement with the observed increase of $q_{\mathrm{CO} 2}$ in W3110vgb (Table 1). In contrast, the expression of $s u c A$ and $s u c B$ was lower in BL21 $v g b^{+}$, relative to its wild-type, in agreement with the $q_{\mathrm{CO} 2}$ decrease on (Table 1). Moreover, the decarboxylation of $\alpha$-ketoglutarate produces NADH. The lower NADH synthesis expected in strain BL21 as effect of $\mathrm{VHb}$ expression, can be linked with a reduced transmembrane proton gradient. As consequence, less ATP is produced (Table 1). $c y o A$ and $c y o B$ code for components of the cytochrome bo $_{3}$, which is the cytochrome prevailing under aerobic conditions in E. coli. The transcription levels of

Table 1. Kinetic and stoichiometric parameters of the wild type and engineered strains.

\begin{tabular}{|c|c|c|c|c|}
\hline Parameter/Strain & W3110 & $\mathrm{W} 3110 v g b^{+}$ & BL21 & BL21 $v g b^{+}$ \\
\hline$\mu\left(\mathrm{h}^{-1}\right)$ & $0.53 \pm 0.01$ & $0.53 \pm 0.02$ & $0.54 \pm 0.00$ & $0.46 \pm 0.02$ \\
\hline$Y_{x / s}\left(\mathrm{~g} \mathrm{~g}^{-1}\right)$ & $0.49 \pm 0.02$ & $0.43 \pm 0.03$ & $0.44 \pm 0.00$ & $0.41 \pm 0.00$ \\
\hline$Y_{\text {acet } / S}\left(\mathrm{~g} \mathrm{~g}^{-1}\right)$ & $0.13 \pm 0.01$ & $0.09 \pm 0.01$ & $0.05 \pm 0.01$ & $0.02 \pm 0.00$ \\
\hline$-q_{S}\left(\mathrm{gg}^{-1} \mathrm{~h}^{-1}\right)$ & $1.10 \pm 0.07$ & $1.31 \pm 0.08$ & $1.23 \pm 0.00$ & $1.13 \pm 0.05$ \\
\hline$q_{\text {acet }}\left(\mathrm{g} \mathrm{g}^{-1} \mathrm{~h}^{-1}\right)$ & $0.16 \pm 0.01$ & $0.10 \pm 0.01$ & $0.05 \pm 0.01$ & $0.02 \pm 0.00$ \\
\hline$q_{l a c}\left(\mathrm{~g} \mathrm{~g}^{-1} \mathrm{~h}^{-1}\right)$ & $0.008 \pm 0.001$ & $0.010 \pm 0.001$ & $0.002 \pm 0.000$ & $0.049 \pm 0.006$ \\
\hline$q_{s u c}\left(\mathrm{~g} \mathrm{~g}^{-1} \mathrm{~h}^{-1}\right)$ & $0.012 \pm 0.001$ & $0.015 \pm 0.001$ & $0.002 \pm 0.000$ & $0.001 \pm 0.000$ \\
\hline$-q_{\mathrm{O} 2}\left(\mathrm{mmol} \mathrm{g}^{-1} \mathrm{~h}^{-1}\right)$ & $15.0 \pm 0.5$ & $18.3 \pm 1.1$ & $19.9 \pm 2.1$ & $15.4 \pm 0.4$ \\
\hline$q_{\mathrm{CO} 2}\left(\mathrm{mmol} \mathrm{g}^{-1} \mathrm{~h}^{-1}\right)$ & $13.1 \pm 0.7$ & $15.9 \pm 1.0$ & $18.3 \pm 1.8$ & $15.0 \pm 0.8$ \\
\hline$q_{\text {ATP }}\left(\mathrm{mmol} \mathrm{g}^{-1} \mathrm{~h}^{-1}\right)^{\mathrm{a}}$ & $44.7 \pm 1.49$ & $54.5 \pm 3.0$ & $59.3 \pm 6.3$ & $45.9 \pm 1.19$ \\
\hline RQ & $0.87 \pm 0.03$ & $0.87 \pm 0.02$ & $0.92 \pm 0.02$ & $0.94 \pm 0.00$ \\
\hline
\end{tabular}

Data correspond to the mean of duplicate cultures and were calculated during the exponential growth phase. $m$ : specific growth rate; $Y_{x / s}$ : biomass yield from glucose; $Y_{\text {acet } / S}$ : acetate yield from glucose; $q_{s}$ : specific glucose consumption rate; $q_{\text {acet }}:$ specific acetate production rate; $q_{\text {lac }}$ : specific lactate production rate; $q_{s u c}:$ specific succinate production rate; $q_{\mathrm{O} 2}$ : specific oxygen uptake rate; $q_{\mathrm{CO} 2}$ : specific carbon dioxide production rate. $q_{\mathrm{ATP}}$ was calculated with an stoichiometry of $1.49 \mathrm{~mol} \mathrm{ATP} /{ }^{1} / 2 \mathrm{molO}_{2}[13]$. 


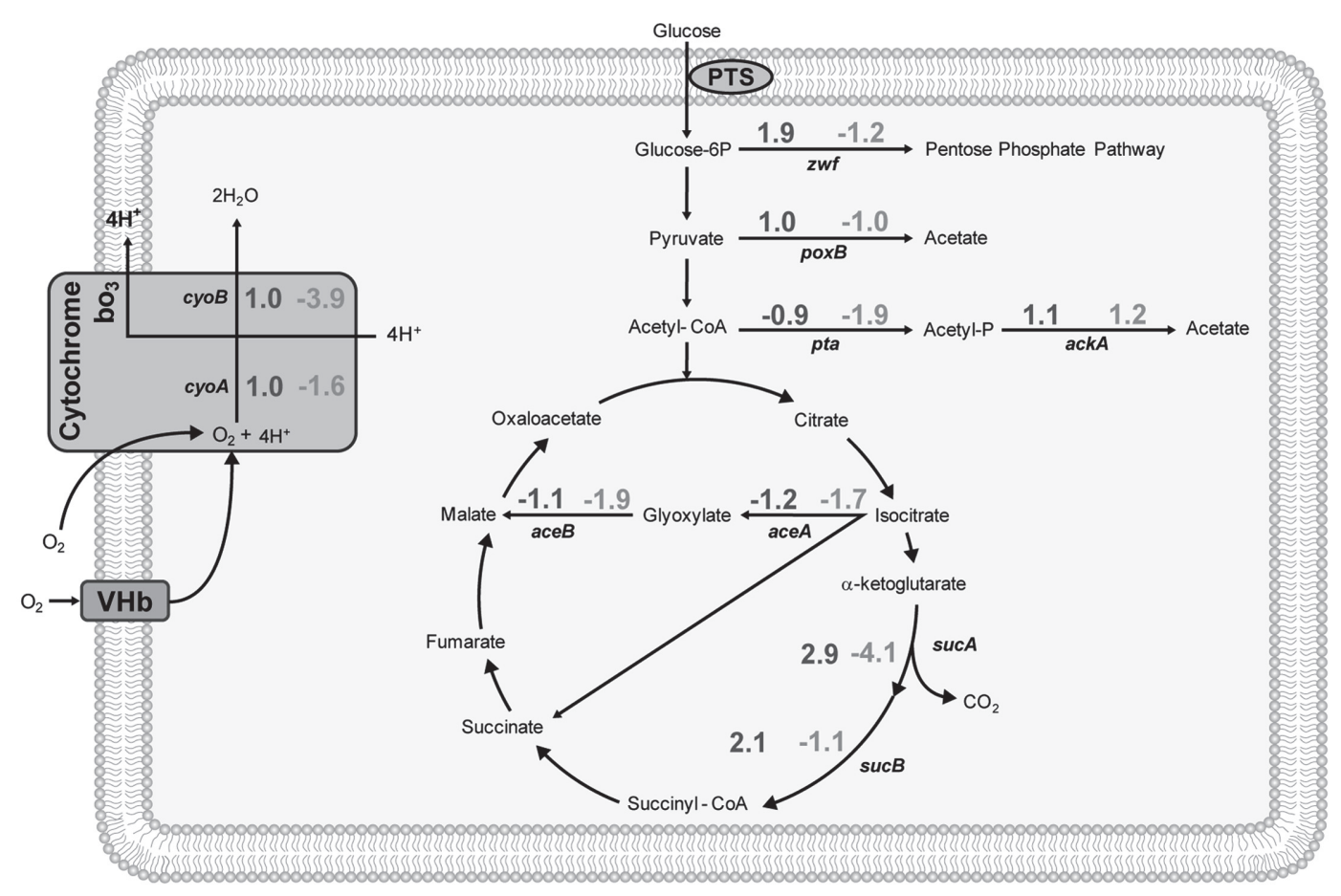

Fig. 3. Expression levels of strains $\mathrm{W}^{3} 110 v g b^{+}$relative to $\mathrm{W} 3110 \mathrm{vg} b^{-}$(left numbers) and $\mathrm{BL} 21 v g b^{+}$relative to BL21 $\mathrm{gg}^{-}$(right numbers). The results are shown above the arrows of the reactions where the transcription product is involved. Standard deviation ranges between 4 and $20 \%$.

such genes were not affected by VHb in W3110. In contrast, $c y o B$ was repressed in BL21. This may be related with a lower NADH regeneration NADH by SucA (1 NADH mol per succinyl-CoA mol synthetized), which is a key cofactor driving the respiratory chain and consistent with the lower $q_{02}$ observed (Table 1). The results show that the effects of aerobic VHb expression will depend on the particular strain used, and that different advantages (like reduced acetate accumulation) and potential disadvantages (like lower growth rate in BL21) may arise.

\section{Materials and Methods}

\section{Strains and Culture Conditions and Off-Line Analyses}

The Escherichia coli strains used in this study were W3110recA and BL21(DE3) recA. The vgb gene, coding for the Vitreoscilla stercoraria hemoglobin was synthesized by GeneScript (USA). Then, it was cloned in pLoxGentrc between the NcoI and EcoRI sites downstream the $\operatorname{trc}$ promoter $\left(\mathrm{P}_{t r c}\right)$. The DNA region comprising $\mathrm{P}_{t r c}, v g b$ and gentamicin resistance genes flanked by Lox sequences was then PCR amplified from the using the high-fidelity polymerase Phusion (ThermoScientific, USA) and oligonucleotides 5'-GTTATTTCTTGATGTCTCTGACCA GACACCCATCAACAGTATTATTTTCTAGCTTATCATCGACTGCACG-3'and 5'-GACATGGCCTGCCCG GTTATTATTATTTTTGACACCAGACCAACTGCACAGATGCGTAAGGAGAA-3' [19, 20]. The resulting $2,487 \mathrm{nt}$ product was used to transform the $E$. coli strains bearing the plasmid was $\mathrm{pKD} 46$, in which the Red system proteins were already induced with arabinose. This way, the PCR product was integrated into the chromosome by homologous recombination between lacI and lacZ. The integration was confirmed by resistance to gentamicin and the absence of blue coloration in colonies grown in LB agar containing X-Gal $10 \mu \mathrm{g} / \mathrm{ml}$ and IPTG (1 mM). PCR tests demonstrated successful integration of $v g b$. Engineered cells were inoculated in $250 \mathrm{ml}$ shake-flasks containing $50 \mathrm{ml}$ of mineral medium during $12-16 \mathrm{~h}$, in orbital shakers at $250 \mathrm{rpm}$ and $37^{\circ} \mathrm{C}$. The composition of the medium is as follows: $\mathrm{K}_{2} \mathrm{HPO}_{4}, 17 ; \mathrm{KH}_{2} \mathrm{PO}_{4}, 5.3 ;\left(\mathrm{NH}_{4}\right)_{2} \mathrm{SO}_{4}, 2.5 ; \mathrm{NH}_{4} \mathrm{Cl}, 1.0 ;$ Citrate- $\mathrm{Na}_{3} \cdot 2 \mathrm{H}_{2} \mathrm{O}, 2$; $\mathrm{MgSO}_{4} \cdot 7 \mathrm{H}_{2} \mathrm{O}, 1.0$; Thiamine- $\mathrm{HCl}, 0.01$. The medium was supplemented with trace element solution $(2 \mathrm{ml} / \mathrm{l})$ and kanamycin sulfate $(50 \mu \mathrm{g} / \mathrm{ml})$. The trace element solution composition (in $\mathrm{g} / \mathrm{l}$ ) was $\mathrm{ZnCl}_{2}, 10.5$; EDTA, 5.5; $\mathrm{CoSO}_{4} \cdot 7 \mathrm{H}_{2} \mathrm{O}, 1.5 ; \mathrm{MnSO}_{4} \cdot \mathrm{H}_{2} \mathrm{O}, 6.4 ; \mathrm{CuSO}_{4} \cdot 5 \mathrm{H}_{2} \mathrm{O}, 1.1 ; \mathrm{H}_{3} \mathrm{BO}_{3}, 1.5 ; \mathrm{Na}_{2} \mathrm{MoO}_{4} \cdot 2 \mathrm{H}_{2} \mathrm{O}, 1 ; \mathrm{FeCl}_{3} \cdot 6 \mathrm{H}_{2} \mathrm{O}, 51.4$; and Cit$\mathrm{H} \cdot \mathrm{H}_{2} \mathrm{O}, 39.9$. The broth was collected and used to inoculate the main cultures. Cultures were performed in $500 \mathrm{ml}$ of medium in a 1-1 Biostat A Plus bioreactor (Sartorius BBI, Germany) at $37^{\circ} \mathrm{C}, \mathrm{pH} 7.2$. Dissolved oxygen tension (DOT) was measured using a polarographic sensor (USA). DOT was controlled at $30 \%$ air sat. by a varying the stirring rate. Off-gas composition was monitored through a BlueInOne Ferm (BlueSens, Germany) gas analyzer. OTR and CTR were calculated with the proper mass balances [20]. Three independent cultures under each condition were performed.

Active VHb was measured by CO-difference spectral analysis as described by Yang et al. [11] from samples taken during the exponential growth phase and lysed with lysozyme. The CO-difference spectra was measured 
with an UV-Vis Evolution 300 Spectrophotometer (Thermo Scientific) using the extinction coefficient $\varepsilon_{(419-436 \mathrm{~nm})}$ $=274 \mathrm{mM}^{-1} \mathrm{~cm}^{-1}$ [11]. Biomass concentration was measured as dry cell weight. Glucose was quantified in an YSI 2700 biochemistry analyzer (YSI Inc., USA). Acetate, lactate and succinate were quantified using enzyme kits (RBiopharm, Germany).

\section{Transcriptional Analysis}

RNA extraction, purification and quality control, as well as cDNA synthesis were performed as previously detailed [20]. RNA integrity numbers of the purified samples were between 7.8 and 9.2. cDNA was synthesized using the primers listed in Supplementary Table 1. The gene gmk (coding for guanylate kinase) was selected from 9 candidates as the internal control gene, due to a nearly constant $C q$ value in all strains (Table S2). RT-qPCR was performed as described before [21], and the transcription levels calculated after Livak and Schmittgen [22]. These experiments were compliant with MIQE guidelines [23].

\section{Acknowledgments}

Financial support from CONACyT grant number 256617 is acknowledged.

\section{Conflict of Interests}

The authors have no financial conflicts of interest to declare.

\section{References}

1. Wei XX, Chen GQ. 2008. Applications of the VHb gene $v g b$ for improved microbial fermentation processes. Methods Enzymol. 436: $273-283$

2. Stark BC, Dikshit KL, Pagilla KR. 2012. The biochemistry of Vitreoscilla hemoglobin. Comput. Struct. Biotechnol. J. 3: e201210002.

3. Stark BC, Pagilla KR, Dikshit KL. 2015. Recent applications of Vitreoscilla hemoglobin technology in bioproduct synthesis and bioremediation. Appl. Microbiol. Biotechnol. 99: 1627-1636.

4. Pablos TE, Sigala JC, Le Borgne S, Lara AR. 2014. Aerobic expression of Vitreoscilla hemoglobin efficiently reduces overflow metabolism in Escherichia coli. Biotechnol. J. 9: 791-799.

5. Juárez M, González-De la Rosa CH, Memún E, Sigala JC, Lara AR. 2017. Aerobic expression of Vitreoscilla hemoglobin improves the growth performance of CHO-K1 cells. Biotechnol. J. 12: 1600438.

6. Zhang H, Kang X, Xiao N, Gao M, Zhao Y, Zhang B, et al. 2019. Intracellular expression of Vitreoscilla haemoglobin improves lipid production in Yarrowia lipolytica. Lett. Appl. Microbiol. 68: 248-257.

7. Marisch K, Bayer K, Scharl T, Mairhofer J, Krempl PM, Hummel K, et al. 2013. Comparative analysis of industrial Escherichia coli K12 and B strains in high-glucose batch cultivations on process-, transcriptome- and proteome level. PLoS One 8: e70516.

8. Monk JM, Koza A, Campodonico MA, Machado D, Seoane JM, Palsson BO, et al. 2016. Multi-omics quantification of species variation of Escherichia coli links molecular features with strain phenotypes. Cell Syst. 3: 238-251.

9. Noronha SB, Yeh HJC, Spande TF, Shiloach J. 2000. Investigation of the TCA cycle and the glyoxylate shunt in Escherichia coli BL21 and JM109 using ${ }^{13} \mathrm{C}-\mathrm{NMR} / \mathrm{MS}$. Biotechnol. Bioeng. 68: 316-327.

10. Phue JN, Noronha SB, Hattacharyya R, Wolfe AJ, Shiloah J. 2005. Glucose metabolism at high density growth of E. coli B and E. coli K: differences in metabolic pathways are responsible for efficient glucose utilization in E. coli B as determined by microarrays and northern blot analyses. Biotechnol. Bioeng. 90: 805-820.

11. Yang J, Webster DA, Stark BC. 2005. ArcA works with Fnr as a positive regulator of Vitreoscilla (bacterial) hemoglobin gene expression in Escherichia coli. Microbiol. Res. 160: 405-415.

12. Tsai PS, Hatzimanikatis V, Bailey JE. 1996. Effect of Vitreoscilla hemoglobin dosage on microaerobic Escherichia coli carbon and energy metabolism. Biotechnol. Bioeng. 49: 139-150.

13. Taymaz-Nikerel H, Borujeni AE, Verheijen PJT, Heijnen JJ, van Gulik WM. 2010. Genome-derived minimal metabolic models for Escherichia coli MG1655 with estimated in vivo respiratory ATP stoichiometry. Biotechnol. Bioeng. 107: 369-381

14. Maharjan RP, Seeto S, Ferenci T. 2007. Divergence and redundancy of transport and metabolic rate-yield strategies in a single Escherichia coli population. J. Bacteriol. 189: 2350-2358.

15. Frey AD, Fiaux J, Szyperski T, Wüthrich K, Bailey JE, Kallio PT. 2001. Dissection of central carbon metabolism of hemoglobinexpressing Escherichia coli by ${ }^{13} \mathrm{C}$ nuclear magnetic resonance flux distribution analysis in microaerobic bioprocesses. Appl. Environ. Microbiol. 67: 680-687.

16. Kim TS, Jung HY, Kim SY, Zhang L, Li J, Sigdel S, et al. 2015. Reduction of acetate and lactate contributed to enhancement of a recombinant protein production in E. coli BL21. J. Microbiol. Biotechnol. 25: 1093-1100.

17. Rowley DL, Fawcett WP, Wolf RE Jr. 1992. Molecular characterization of mutations affecting expression level and growth ratedependent regulation of the Escherichia coli zwf gene. J. Bacteriol. 174: 623-626.

18. Kim HU, Kim WJ, Lee SY. 2013. Flux-coupled genes and their use in metabolic flux analysis. Biotechnol. J. 8: 1035-1042.

19. Jaén KE, Velázquez D, Delvigne F, Sigala JC, Lara AR. 2019b. Engineering E. coli for improved microaerobic pDNA production. Bioproc. Biosyst. Eng. 42: 1457-1466.

20. Jaén KE, Velázquez D, Sigala JC, Lara AR. 2019a. Design of a microaerobically inducible replicon for high-yield plasmid DNA production. Biotechnol. Bioeng. 116: 2514-2525.

21. Sabido A, Sigala JC, Hernández-Chávez G, Flores N, Gosset G, et al. 2014. Physiological and transcriptional characterization of Escherichia coli strains lacking interconversion of phosphoenolpyruvate and pyruvate when glucose and acetate are coutilized. Biotechnol. Bioeng. 111: 1150-1160.

22. Livak KJ, Schmittgen TD. 2001. Analysis of relative gene expression data using real-time quantitative PCR and the $2^{-\Delta \Delta C T}$ method. Methods 25: 402-408.

23. Bustin SA, Benes V, Garson JA, Hellemans J, Huggett J, Kubista M, et al. 2009. The MIQE guidelines: minimum information for publication of quantitative real-time PCR experiments. Clin. Chem. 55: 611-622. 